\title{
Excluded-Volume Effect on Dipole Moment of Polar Macromolecules
}

\author{
Masao Dor \\ Department of Applied Physics, Faculty of Engineering, \\ University of Tokyo, Bunkyo-ku, Tokyo, Japan.
}

(Received September 20, 1971)

\section{KEY WORDS Excluded Volume / Dipole Moment / End-to-end \\ Vector / Exact Scaling Relation /}

Recently Nagai and Ishikawa have investigated the excluded volume effect on the dipole moment of a polymer chain by the conventional perturbation method. They found that, up to the term in $z^{3}$ ( $z$, the excluded-volume parameter), the following simple relation exists, and they presumed that the relation may hold for any other higher order of perturbation.

$$
\frac{\left\langle\boldsymbol{u}^{2}\right\rangle}{\left\langle\boldsymbol{u}^{2}\right\rangle_{0}}-1=\frac{\langle\boldsymbol{r} \cdot \boldsymbol{u}\rangle_{0}{ }^{2}}{\left\langle\boldsymbol{r}^{2}\right\rangle_{0}\left\langle\boldsymbol{u}^{2}\right\rangle_{0}}\left(\frac{\left\langle\boldsymbol{r}^{2}\right\rangle}{\left\langle\boldsymbol{r}^{2}\right\rangle_{0}}-1\right)
$$

In eq $1, r$ and $u$ are the end-to-end vector and the dipole moment vector of the chain respectively, and the average with and without subscript 0 refer to those in the absence and in the presence of the interaction between segments.

We will show in this paper that Nagai and Ishikawa's presumption is actually valid not only for any order of perturbation but also for any type of interaction and, in addition, there exists a more general scaling relation between the distribution function of $\boldsymbol{r}$ and $\boldsymbol{u}$.

The distribution function of a polymer conformation can be written in the form,

$$
\begin{aligned}
& \boldsymbol{P}\left(\boldsymbol{u}, \boldsymbol{v}_{1}, \boldsymbol{v}_{2}, \cdots \boldsymbol{v}_{N}\right) \\
= & \boldsymbol{P}_{0}\left(\boldsymbol{u}, \boldsymbol{v}_{1}, \boldsymbol{v}_{2}, \cdots \boldsymbol{v}_{N}\right) Q\left(\boldsymbol{v}_{1}, \boldsymbol{v}_{2}, \cdots \boldsymbol{v}_{N}\right) .
\end{aligned}
$$

In eq $2, \boldsymbol{v}_{i}$ is the end-to-end vector of $i$-th segment, $N$ is the number of segments in the chain and $P_{0}$ is the unperturbed distribution function, the explicit form of which will be given later. In the case of pairwise interaction, $Q$ is given by

$$
Q=\exp \left[-\frac{1}{k T} \sum_{l<m} U\left(\boldsymbol{v}_{l}+\boldsymbol{v}_{l+1}+\cdots+\boldsymbol{v}_{m}\right)\right],
$$

where $U$ is the the interaction potential between segments. The particular form of $Q$, however, does not affect the results and the following argument applies to any type of interaction. From eq 2 we can derive the distribution function of $\boldsymbol{r}$ and $\boldsymbol{u}$,

$$
\begin{gathered}
\boldsymbol{P}_{u}(\boldsymbol{u})=\int \mathrm{d}^{3} \boldsymbol{v}_{1} \cdots \mathrm{d}^{3} \boldsymbol{v}_{N} P\left(\boldsymbol{u}, \boldsymbol{v}_{1}, \cdots \boldsymbol{v}_{N}\right) . \\
\boldsymbol{P}_{r}(\boldsymbol{r})=\int \mathrm{d}^{3} \boldsymbol{u} \mathrm{d}^{3} \boldsymbol{v}_{1} \cdots \mathrm{d}^{3} \boldsymbol{v}_{N} \\
\quad \times \delta\left(\boldsymbol{v}_{1}+\cdots+\boldsymbol{v}_{N}-\boldsymbol{r}\right) \boldsymbol{P}\left(\boldsymbol{u}, \boldsymbol{v}_{1}, \cdots \boldsymbol{v}_{N}\right)
\end{gathered}
$$

Here we use the Fourier transform for each distribution function. For arbitrary function $F\left(\boldsymbol{r}_{1}, \boldsymbol{r}_{2}, \cdots \boldsymbol{r}_{n}\right)$ the Fourier transform function is denoted by $\widetilde{F}\left(\boldsymbol{k}_{1}, \boldsymbol{k}_{2}, \cdots \boldsymbol{k}_{n}\right)$.

$$
\begin{aligned}
\tilde{F}\left(\boldsymbol{k}_{1}, \boldsymbol{k}_{2}, \cdots \boldsymbol{k}_{n}\right)= & \int \mathrm{d}^{3} \boldsymbol{r}_{1} \cdots \mathrm{d}^{3} \boldsymbol{r}_{n} F\left(\boldsymbol{r}_{1}, \boldsymbol{r}_{2} \cdots \boldsymbol{r}_{n}\right) \\
& \times \mathrm{e}^{i\left(\boldsymbol{k}_{1} \cdot \boldsymbol{r}_{1}+\cdots+\boldsymbol{k}_{n} \boldsymbol{r}_{n}\right)} .
\end{aligned}
$$

Then eq 2, 4, and 5 are transformed into,

$$
\begin{aligned}
& \widetilde{P}\left(\boldsymbol{k}_{u}, \boldsymbol{k}_{1}, \cdots \boldsymbol{k}_{N}\right)= \int \frac{\mathrm{d}^{3} q_{1}}{(2 \pi)^{3}} \cdots \frac{\mathrm{d}^{3} q_{N}}{(2 \pi)^{3}} \\
& \times \widetilde{P}\left(\boldsymbol{k}_{u}, \boldsymbol{k}_{1}-\boldsymbol{q}_{1}, \cdots, \boldsymbol{k}_{N}-\boldsymbol{q}_{N}\right) \\
& \times \widetilde{Q}\left(\boldsymbol{q}_{1}, \cdots, \boldsymbol{q}_{N}\right) \\
& \widetilde{P}_{u}\left(\boldsymbol{k}_{u}\right)=\widetilde{P}\left(\boldsymbol{k}_{u}, \boldsymbol{k}_{1}=\boldsymbol{k}_{2}=\cdots=\boldsymbol{k}_{N}=0\right) \\
& \widetilde{\boldsymbol{P}}_{r}\left(\boldsymbol{k}_{r}\right)=\widetilde{\boldsymbol{P}}\left(\boldsymbol{k}_{u}=0 \quad \boldsymbol{k}_{1}=\boldsymbol{k}_{2}=\cdots=\boldsymbol{k}_{N}=\boldsymbol{k}_{r}\right)
\end{aligned}
$$

According to Nagai and Ishikawa we assume that $\boldsymbol{P}_{0}$ is a multi-variate Gaussian function and can be characterized by three parameters, $C_{r r}$, $C_{r u}$, and $C_{u u}$.

$$
\begin{gathered}
C_{r r}=\lim _{N \rightarrow \infty} N^{-1}\left\langle\boldsymbol{r}^{2}\right\rangle_{0}, \quad C_{r u}=\lim _{N \rightarrow \infty} N^{-1}\langle\boldsymbol{r} \cdot \boldsymbol{u}\rangle_{0}, \\
C_{u u}=\lim _{N \rightarrow \infty}\left\langle\boldsymbol{u}^{2}\right\rangle_{0}
\end{gathered}
$$


Then we can write $\widetilde{P}_{0}$ explicitly.

$$
\begin{aligned}
& \widetilde{P}_{0}\left(\boldsymbol{k}_{u}, \boldsymbol{k}_{1}, \cdots, \boldsymbol{k}_{N}\right)=\exp \left[-\frac{1}{6}\left(N C_{u u} \boldsymbol{k}_{u}{ }^{2}\right.\right. \\
& \left.\left.\quad+2 \sum_{l=1}^{N} C_{r u} \boldsymbol{k}_{u} \cdot \boldsymbol{k}_{l}+\sum_{l=1}^{N} C_{r r} k_{l}{ }^{2}\right)\right]
\end{aligned}
$$

Thus we have the following relation:

$$
\begin{aligned}
\widetilde{P}_{0}\left(\boldsymbol{k}_{u},-\boldsymbol{q}_{1},-\boldsymbol{q}_{2},-\boldsymbol{q}_{N}\right) \\
=\exp \left[-\frac{N}{6}\left(C_{u u}-\frac{C_{r u}^{2}}{C_{r r}}\right) \boldsymbol{k}_{u}{ }^{2}\right] \\
\times \widetilde{P}_{0}\left(\boldsymbol{k}_{u}=0, \frac{C_{r u}}{C_{r r}} \boldsymbol{k}_{u}-\boldsymbol{q}_{1}, \frac{C_{r u}}{C_{r r}} \boldsymbol{k}_{u}-\boldsymbol{q}_{2},\right. \\
\left.\quad \cdots, \frac{C_{r u}}{C_{r r}} \boldsymbol{k}_{u}-\boldsymbol{q}_{N}\right)
\end{aligned}
$$

From eq 7-9 and using the relation of eq 12 we obtain the following scaling relation.

$$
\begin{aligned}
\widetilde{\boldsymbol{P}}_{u}\left(\boldsymbol{k}_{u}\right)= & \exp \left[-\frac{N}{6}\left(C_{u u}-\frac{C_{r u}^{2}}{C_{r r}}\right) \boldsymbol{k}_{u}{ }^{2}\right) \\
& \times \widetilde{\boldsymbol{P}}_{r}\left(\frac{C_{r u}}{C_{r r}} \boldsymbol{k}_{u}\right)
\end{aligned}
$$

Using the following relations:

$$
\begin{aligned}
& \left\langle\boldsymbol{u}^{2}\right\rangle=-\left.6 \frac{\partial}{\partial \boldsymbol{k}_{u}{ }^{2}} \log \widetilde{P}_{u}\left(\boldsymbol{k}_{u}\right)\right|_{\boldsymbol{k}_{u}=0} \\
& \left\langle\boldsymbol{r}^{2}\right\rangle=-\left.6 \frac{\partial}{\partial \boldsymbol{k}_{r}{ }^{2}} \log \widetilde{P}_{r}\left(\boldsymbol{k}_{r}\right)\right|_{\boldsymbol{k}_{r}=0}
\end{aligned}
$$

we immediately obtain eq 1 . Here it should be noted that eq 10 is valid only if we ignore the contribution of the order of $1 / N$. The present argument, therefore, cannot apply to the case where the dipole moment is independent of degree of polymerization. ${ }^{2}$

Note. The proof of eq 12 in Nagai and Ishikawa's paper will be given in the following. Since the following inequality holds for arbitrary real number $t$,

$$
\left\langle(\boldsymbol{r}+t \boldsymbol{u})^{2}\right\rangle_{0}=\left\langle\boldsymbol{u}^{2}\right\rangle_{0} t^{2}+2\langle\boldsymbol{r} \cdot \boldsymbol{u}\rangle_{0} t+\left\langle\boldsymbol{r}^{2}\right\rangle_{0} \geqq 0
$$

the following inequality must hold:

$$
\left\langle\boldsymbol{r}^{2}\right\rangle_{0}\left\langle\boldsymbol{u}^{2}\right\rangle_{0} \geqq\langle\boldsymbol{r} \cdot \boldsymbol{u}\rangle_{0}^{2}
$$

Of course the average in eq 17 may be of a quite general nature in so far as eq 16 is satisfied.

Acknowledgement. The author wishes to thank Professor Koji Okano and Dr. Yoshikazu Tanabe for their helpful advice.

\section{REFERENCES}

1. K. Nagai and T. Ishikawa, Polymer J., 2, 416 (1971).

2. K. Okano and Y. Tanobe, Preprints, SPSJ, 16th Symposium on Macromolecules, Japan, Fukuoka, 1967, p 440. 